\title{
Dependence of equivalent width of quasar emission lines on UV-optical spectral index
}

\author{
Olena Torbaniuk ${ }^{1,3}$ and Ganna Ivashchenko ${ }^{2,3}$ \\ ${ }^{1}$ Faculty of Physics, Taras Shevchenko National University of Kyiv, \\ Glushkova ave. 4, 03127 Kyiv, Ukraine \\ email: el.torbaniuk@gmail.com \\ ${ }^{2}$ Astronomical Observatory, Taras Shevchenko National University of Kyiv, \\ Observatorna str. 3, 04058 Kyiv, Ukraine \\ email: g.ivashchenko@gmail.com \\ ${ }^{3}$ Main Astronomical Observatory of the National Academy of Sciences of Ukraine, \\ Akademika Zabolotnoho str. 27, 03680 Kyiv, Ukraine
}

\begin{abstract}
Using 192 composite spectra stacked from subsamples of individual SDSS quasar spectra binned in spectral index, $\alpha_{\lambda}$, and logarithm of monochromatic luminosity at $1450 \AA$, $\log l_{1450}$, and modelling separate spectral ranges with superposition of emission lines it was found that: there is a dependence of emission line equivalent width (EW) on $\alpha_{\lambda}$ (correlation or anti-correlation) for some lines, mostly for those lines for which the Baldwin effect is detected, while for the lines for which the Baldwin effect is not observed any $\alpha_{\lambda}$-dependence was found.
\end{abstract}

Keywords. quasars: emission lines, quasars: general

\section{Introduction}

Despite of general similarity in UV-optical SED of quasars, which allows to use their composite (mean) spectra for various studies, they differ in equivalent width (EW) of emission lines and spectral index $\alpha_{\lambda}$. Continuum and emission lines are believed to originate from the hot accretion disc and circumnuclear fast moving clumps, correspondingly. The proximity of these regions is considered to be the most promising explanation of the Baldwin (1977) effect: the inverse correlation of EW of some emission lines with the monochromatic luminosities at UV region, e.g., for Ly $\alpha(1215 \AA)$, Siri $(1260 \AA)$,

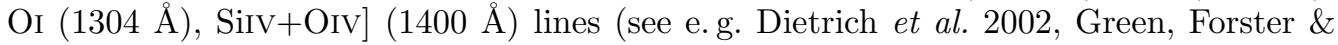
Kuraszkiewicz 2001, and Shang et al. 2003). But the physical explanation of the difference in $\alpha_{\lambda}$ and relation of quasar luminosity and lines' EW to $\alpha_{\lambda}$ are still not clear.

Previously, using composite spectra of quasars with different $\alpha_{\lambda}$, we showed that there is no correlation between $\alpha_{\lambda}$ and $\log l_{1450}$ (Ivashchenko, Sergijenko \& Torbaniuk 2013), and did not found any dependence of EW of lines within 1210-1450 $\AA$ on spectral index $\alpha_{\lambda}$ (calculated within the range 1270-1480 $\AA$ ) (Torbaniuk, Ivashchenko \& Sergijenko 2012). But due to the fact, that the latter study was carried out without separation by luminosity, its result requires verification on a sample of spectra with different luminosity as well. Such verification has been done in the present study.

\section{The data and method}

For our study we stacked $16 \times 12=192$ composite spectra from spectra subsamples binned in $\alpha_{\lambda}=-2.3+i \cdot 0.1(i=0.15)$ and $\log l_{1450}=42.2+k \cdot 0.1(k=0.11)$, using a sample of quasar spectra selected from the Sloan Digital Sky Survey (SDSS) Data Release 7 by Ivashchenko, Sergijenko \& Torbaniuk (2013). 
The wavelength ranges 1215-1285 $\AA, 1290-1320 \AA, 1320-1350 \AA$ and 1350-1430 were considered separately and fitted with the help of the IDL lmfit subroutine with a superposition of constant or power-law continuum and the smallest possible number of Gaussian-profile emission lines. Then the EWs of these four separate superpositions of lines were calculated using obtained model parameters.

\section{Results}

The obtained $\alpha_{\lambda}-\mathrm{EW}$ diagrams for different luminosities showed the presence of correlation or anti-correlation between $\alpha_{\lambda}$ and EW for those lines, for which the Baldwin effect is seen. The significance of these results were checked with the F-test using constant and linear models as restricted and unrestricted ones, respectively. The more detailed descriptions of each case are presented below.

(i) For lines $\mathrm{Ly} \alpha+\mathrm{OV}+\mathrm{NV}+\mathrm{SiII}^{*}+\mathrm{SiII}(1215-1285 \AA)$ the EW decreases with $\alpha_{\lambda}$; the significance of the linear coefficient of regression in most cases is $\sim 2 \sigma$, and the Baldwin effect is clearly seen for these set of lines. The more detailed analysis with separation of this region into three parts showed the same trend for Ly $\alpha+$ Ov and SiII, unlike $\mathrm{NV}+\mathrm{SiI}^{*}$ for which we did not found any dependence. At the same time, the Baldwin effect is seen for Ly $\alpha+\mathrm{Ov}$ and SirI lines, while for $\mathrm{Nv}+\mathrm{SiI}^{*}$ we do not see any correlation between EW and luminosity. Note, that the presence of the Baldwin effect was found previously both for $\mathrm{Ly} \alpha+\mathrm{OV}+\mathrm{NV}+\mathrm{SiII}^{*}$ set of lines and SiI line.

(ii) For lines SiII** + OI + SiII (1290-1320 ̊) the significance of the linear coefficient of regression is $<1 \sigma$, that confirms the absence of any dependence of EW on $\alpha_{\lambda}$ seen by eye. We also did not see any Baldwin effect for this set of lines, although it was found by Shang et al. (2003) for Oi line.

(iii) Unlike the first case, for two other sets of lines, CII + OV + CaII (1320-1350 ^) and X1 + SiIV + OIV] + X2 (1350-1430 ̊), we found the increase of EW with $\alpha_{\lambda}$, but only for the second set we can clearly see the Baldwin effect, found previously by other authors. Here X1 and X2 are two bumps in this set of lines which were not identified.

\section{Acknowledgments}

This work has been supported by the Complex Programme of Scientific Space Studies of the NAS of Ukraine for 2013-2016 and by the Swiss National Science Foundation grant SCOPE IZ7370-152581. The authors are also thankful to the SDSS team.

\section{References}

Baldwin, J. A. 1977, ApJ, 214, 679

Dietrich, M., Hamann, F., Shields, J. C., Constantin, A., Vestergaard, M., Chaffee, F., Foltz, C. B., \& Junkkarinen, V. T. 2002, ApJ, 581, 912

Green, P. J., Forster, K., \& Kuraszkiewicz, J. 2001, ApJ, 556, 727

Ivashchenko, G., Sergijenko, O. \& Torbaniuk O. 2013, MNRAS, DOI:10.1093/mnras/stt2137

Shang, Z., Wills, B. J., Robinson, E. L., Wills, D., Laor, A., Xie, B., \& Yuan, J. 2003, ApJ, 586, 52

Torbaniuk, O., Ivashchenko, G., \& Sergijenko, O. 2012, in: J. Safrankova \& J. Pavlu (eds.), WDS'12 Proc. of Contributed Pap.: Part III - Physics (Prague: Matfyzpress), p. 123 